\title{
3D printing new direction and collaboration in scientific research. A scientometric study using Web of Science, Clarivate Analytics database
}

\author{
Raluca Marinescu (Repanovici)* and Anişor Nedelcu \\ Transylvania University of Braşov, Department of Engineering and Industrial Management, Mihai \\ Viteazul 5, Braşov, Romania
}

\begin{abstract}
A scientometric study to quantify the global research activity in the field of 3D printing has been conducted by the authors. Primary data was acquired as plain text files from Web of Science database (WoS). 11529 results were found. Hereby, the total research productivity, scientific output of countries, individual institution authors, journals and their collaborative networks were assessed. The results - keywords based on co-occurrence and co-citation networks - were visualized by using VOS Viewer, a software tool for constructing and visualizing bibliometric networks.
\end{abstract}

\section{Introduction}

$3 \mathrm{D}$ printing (3DP) is an additive manufacturing process and involves the whole procedure of making 3D solid objects from digital files. Until 2009, 3DP was mostly limited to industrial uses. Originally used as an affordable way to create prototypes for product development within certain industries, 3D printing is becoming more prevalent in the general public. 3D printing is used by car manufacturers, doctors, dentists, prosthetics, aircraft manufacturers, aerospace companies, product designers, architects, engineers and others. At the time of writing, the range of $3 \mathrm{D}$ printed objects varies from simple prototypes to clothing, jewelleries, furniture, homes, drones, vehicles, and even human and animal prosthetics [1]. The use of 3DP in education opens up new approaches and opportunities to students and educators. Being used to develop design skills and creativity, digital fabrication technologies can be used in research to produce scale models and to explore new techniques [2].

Scientometrics is the study of measuring and analyzing science, technology and innovation. Defined as developing "the quantitative methods of the research on the development of science as an informational process" [3], scientometrics include practices of measuring research quality and impact, understanding the process of scientific citations, mapping scientific fields and the use of indicators in research policy and management contexts. As scientometrics can study many aspects of the dynamics of science and technology, the researches introduced tools and applications for science mapping and visualization of bibliometric networks, concepts, current state of the field and future research direction $[4,5]$.

\footnotetext{
*Corresponding author: raluca-maria.marinescu@unitbv.ro
} 
By using scientometrics, the global research trends in 3D printing from 1983-2018 was evaluated and the results found were reported in this paper.

\section{Methodology and source of data}

The required data was collected from Web of Science database for a period of 35 years [6]. 11529 bibliographic records were retrieved on 3D printing in Engineering Fabrication field. The downloaded bibliographic records were analyzed by using VOS Viewer, a software tool for constructing and visualizing bibliometric networks. These networks may for instance include journals, researchers, or individual publications, and they can be constructed based on citation, bibliographic coupling, co-citation, or co-authorship relations. VOS Viewer also offers text mining functionality that can be used to construct and visualize co-occurrence networks of important terms extracted from a body of scientific literature [7].

\section{Data analysis}

From 1983-2018, a total number of 11529 bibliographic publications were published on 3D printing globally. The year-wise publication of research on $3 \mathrm{D}$ printing research productivity is presented in Table 1.

The highest number of publications 3275 (28.4\%) was published in 2017, followed by $3016(26.16 \%)$ in 2016, $1860(16.13 \%)$ in 2015 and $1035(8.98 \%)$ in 2014. The lowest number of publications is $1(0.0087 \%)$ in 1983 [1]. The publication of these records (11529) has been achieved progressively since 1983, when the earliest paper describing 3D printing, "3D - Profile Detection of Etched Patterns Using a Laser Scanner (3D - Scan Detection) for Automatic Inspection of Printed-Circuit Boards" by Ando, M.; Mita, K.; Kakinoki, Y.; et al., was published in Proceedings of The Society of Photo - Optical Instrumentation Engineers, volume 389, pages 44-52 [7].

The global scientific output in 3D printing doubled for the first time in 2014, when the publication's records has been increased from 483 in 2013 to 1035 in 2014 (Table 1). Two years later, in 2016, the global scientific output in 3D printing doubled again, when the record of publications has been increased from 1860 in 2015 to 3016 in 2016.

Table 1. Frequency Distribution of Literature on 3D Printing [7].

\begin{tabular}{c|c|c|c|c|c|c|c} 
Year & Records & Year & Records & Year & Records & Year & Records \\
\hline 2017 & 3275 & 2010 & 174 & 2004 & 56 & 1994 & 10 \\
\hline 2016 & 3016 & 2009 & 164 & 2000 & 45 & 1996 & 8 \\
\hline 2015 & 1860 & 2008 & 143 & 2002 & 41 & 1993 & 6 \\
\hline 2014 & 1035 & 2007 & 135 & 2001 & 40 & 1995 & 5 \\
\hline 2013 & 483 & 2005 & 86 & 1997 & 31 & 1991 & 2 \\
\hline 2012 & 287 & 2006 & 81 & 1999 & 28 & 1992 & 2 \\
\hline 2018 & 224 & 2003 & 66 & 1998 & 23 & 1983 & 1 \\
\hline 2011 & 202 & & & & & &
\end{tabular}

The distribution of 3DP publications by research area is shown in Table 2. Out of 11529 publications, 4574 (39.67\%) records are published in engineering area, followed by material science area with $2620(22.73 \%)$ publications.

Table 2. Research Areas-wise Distribution of Publications in 3D Printing [7].

\begin{tabular}{|c|c|c|}
\hline Research Areas & Record Count & $\%$ of 11529 \\
\hline Engineering & 4574 & $39.674 \%$ \\
\hline Materials Science & 2620 & $22.725 \%$ \\
\hline Science Technology Topics & 1309 & $11.354 \%$ \\
\hline
\end{tabular}




\begin{tabular}{|c|c|c|}
\hline Physics & 1248 & $10.825 \%$ \\
\hline Chemistry & 1085 & $9.411 \%$ \\
\hline Computer Science & 1068 & $9.264 \%$ \\
\hline Optics & 859 & $7.451 \%$ \\
\hline Instruments Instrumentation & 416 & $3.608 \%$ \\
\hline Radiology Nuclear Medicine Medical Imaging & 400 & $3.470 \%$ \\
\hline $\begin{array}{lll} & \text { Surgery }\end{array}$ & 353 & $3.062 \%$ \\
\hline
\end{tabular}

The obtained results were refined and 834 bibliographic records were retrieved on 3D printing in Engineering Manufacturing field.

\section{Results}

The Web of Science database was downloaded as ".txt" file and then it was exported to VOS Viewer for preparing the bibliographic coupling.

The results of the scientific collaboration of countries is shown in Figure 1. This image has been extracted from VOS Viewer software and illustrates the cluster density visualization of the network in 35 years. The density of the network is represented by color variation: the red color indicates the highest density, followed by orange, yellow, green and blue. The results showed that USA is the world leader in scientific papers publishing in $3 \mathrm{D}$ printing field, and cluster analysis showed that the network is formed from 8 different clusters [7]:

- C1: Brazil, France, Germany, Russia

- C2: Denmark, England, Japan, Switzerland

- C3: Australia, India, Malaysia, Wales

- C4: Belgium, Netherlands, China

- C5: Italy, Portugal, Spain

- C6: South Africa, Taiwan, United States of America (USA)

- C7: Greece, South Korea

- C8: New Zealand, Singapore

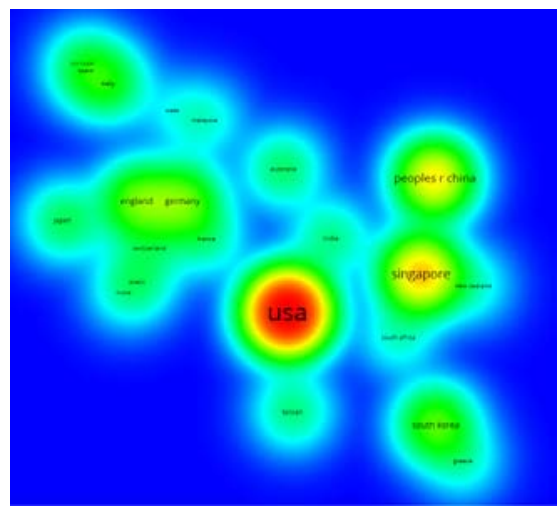

Fig. 1. The density map of countries [7]

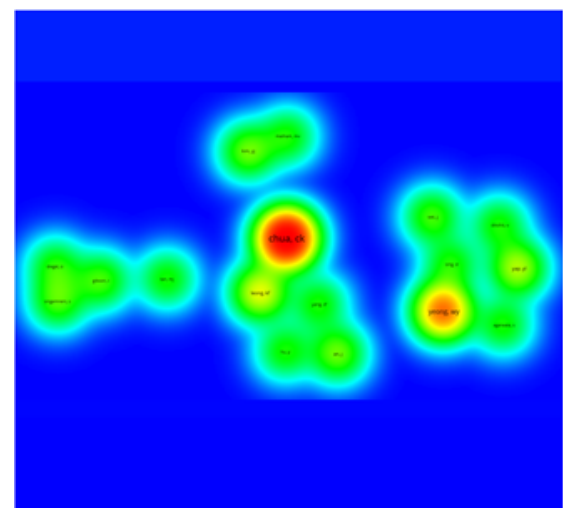

Fig. 2. The density map of authors [7].

Additionally, the results showed that the most productive author is C.K. Chua (Figure 2), and the highest scientific impact (Figure 3, Figure 5) was achieved by Additive Manufacturing Technologies: Rapid Prototyping to Direct Digital Manufacturing, I. Gibson, 2010 publication, followed by $3 D$ Printing and Additive Manufacturing: Principles and Applications, the fourth Edition of Rapid Prototyping, C. K. Chua and K. F. Leong, 2015 publication (Figure 4, Figure 5) [7]. 


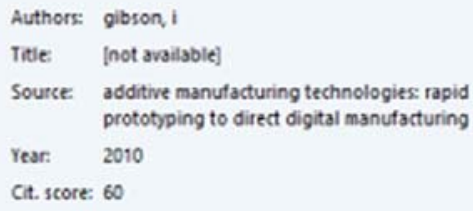

Fig. 3. Scientific impact of publications [7].

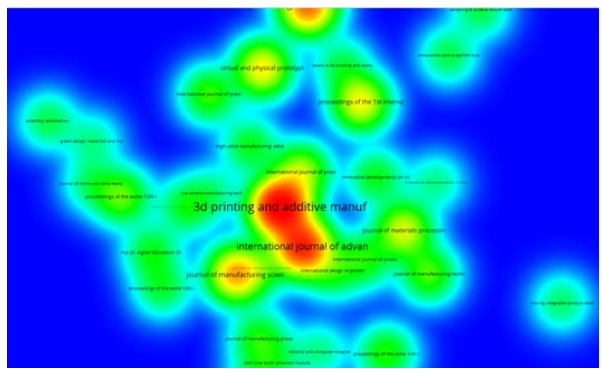

Fig. 5. The density map of publications [7].

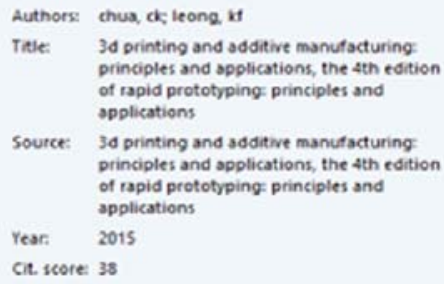

Fig. 4. Scientific impact of publications [7].

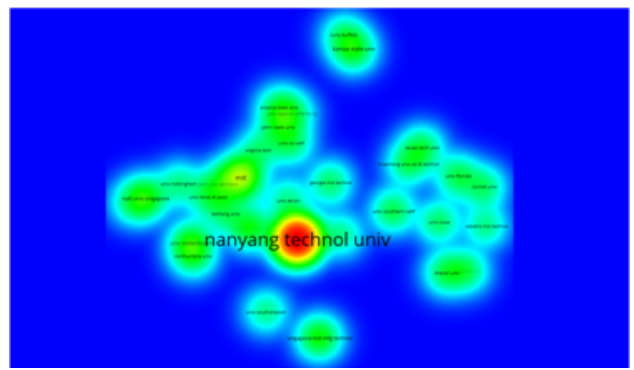

Fig. 6. The density map of institutions [7].

Furthermore, the study reveals that the institution with the highest scientific impact is Nanyang Technological University (Figure 6), and cluster analysis showed 6 collaboration clusters between the universities [7]:

- C1: Auckland University of Technology, Beihang University, Massachusetts Institute of Technology, National University of Singapore, Northumbria University, Seoul National University, Technical University of Denmark, University of Akron, University of Nottingham, Stellenbosch University, University of Texas at El Paso

- C2: Cornell University, Drexel University, Huazhong University of Science and Technology, Stevens Institute of Technology, Texas Tech University, Tsinghua University, University of Florida

- C3: Arizona State University, Georgia Institute of Technology, Pennsylvania State University, University of Applied Sciences Offenburg, University of South California, Virginia Tech University

- C4: Nanyang Technological University, Singapore Institute of Manufacturing Technology, University of Southampton

- C5: University of Illinois, University of Iowa, University of Southern California

- C6: Kansas State University, University at Buffalo

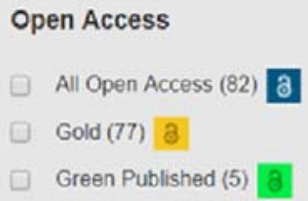

Fig. 7. Screenshot of Open Access Publications [7]. 
Out of 82 open access articles published in 3D printing field, 77 are published as gold open access, and only five articles are published as green open access (Figure 7). Green and gold open access refer to research output published through the traditional model, in which papers are peer-reviewed and then published in journals. All open articles are freely available to read, but there are two routes into open access: gold access, when the research article is made open access by the publisher, being permanently accessible for everyone, immediately after publication, and green access, when the author independently deposits the research outputs in an open access repository.

The study reveals that major source of publication in $3 \mathrm{D}$ printing is conference proceedings papers. The conference proceedings with the highest scientific impact are shown in Table 3 [7].

Table 3. Top Nine Conference Proceedings [7].

\begin{tabular}{|c|c|c|}
\hline Conference Title & $\begin{array}{l}\text { Record } \\
\text { Count }\end{array}$ & $\%$ of 44 \\
\hline $\begin{array}{r}\text { Proceedings of the } 2^{\text {nd }} \text { International Conference on Progress in } \\
\text { Additive Manufacturing PRO AM } 2016\end{array}$ & 12 & $27.273 \%$ \\
\hline $\begin{array}{r}\text { Proceedings of the International Conference on Progress in Additive } \\
\text { Manufacturing }\end{array}$ & 12 & $27.273 \%$ \\
\hline Lasers in 3D Printing and Manufacturing & 7 & $15.909 \%$ \\
\hline $\begin{array}{r}\text { Proceedings of the } 1^{\text {st }} \text { International Conference on Progress in } \\
\text { Additive Manufacturing }\end{array}$ & 7 & $15.909 \%$ \\
\hline World Scientific Series in 3D Printing & 7 & $15.909 \%$ \\
\hline Virtual and Physical Prototyping & 6 & $13.636 \%$ \\
\hline 3D Printing and Additive Manufacturing Principles and Applications & 4 & $9.091 \%$ \\
\hline $\begin{array}{l}\text { 3D Printing and Additive Manufacturing Principles and Applications } \\
\text { the } 4^{\text {th }} \text { Edition of Rapid Prototyping Principles and Applications }\end{array}$ & 4 & $9.091 \%$ \\
\hline Innovative Developments on Virtual and Physical Prototyping & 2 & $4.545 \%$ \\
\hline
\end{tabular}

A recently published summary of $3 \mathrm{D}$ bioprinting publications indexed in Scopus and WoS reveals the 10 most productive nations in terms of 3D bioprinting publishing. Here, the predominant countries are USA with 1491 documents, followed by China with 744 documents and Germany, which holds the third position, with 377 documents [8].

\section{Conclusions and discussion}

This study investigates the research productivity in 3D printing over 35-years, from 1983 to 2018. By using data undertaken from 834 papers that were published in the manufacturing engineering field, the study concludes with the following remarks:

- The maximum number of publications was 3275 in 2017 and the first paper describing 3DP was published in 1983.

- $3 \mathrm{D}$ printing in the manufacturing engineering field has a large geographical coverage (USA, Europe, Asia, Africa), but the collaboration network of countries comprises 8 clusters with 25 countries.

- United States of America is the world leader in scientific publications in 3D printing field, but Nanyang Technological University from Singapore is the institution with the highest scientific impact in this field.

- C.K. Chua is the most productive author by now.

- "Additive Manufacturing Technologies: Rapid Prototyping to Direct Digital Manufacturing" (I. Gibson, 2010) publication achieved the highest scientific impact.

- Nanyang Technological University is the institution with the highest scientific impact. 
- The Proceedings papers were predominant in 3D printing research.

It is proven that academic productivity is a function of multidimensional combination of the academic researcher's work: the scientific work, education and external relationships [9]. Science mapping is a spatial representation of how disciplines, fields, specialties, documents and authors are related to each other. By using scientometrics, researchers can identify new and relevant challenges in their field of research [10].

Additive manufacturing (3D printing), rapid prototyping and reverse engineering technologies are increasingly becoming more popular, being used by engineers, designers, doctors and others. In the last few years, 3D printing industry is witnessing an impressive growth worldwide, but at the time of writing, Romania has no significant visibility and no significant scientific impact in this area [6].

The paper maps the perspective of $3 \mathrm{D}$ printing and explore the hotspots and emerging trends of this field. By using large databases, this study can be used in more areas, as a scientific research method.

\section{References}

1. What is 3D Printing? The definitive guide to additive manufacturing, https://www.3dhubs.com/what-is-3d-printing; Accessing date: 20.01.2018

2. G. Celani. NNJ 14, 3 (2012)

3. V. V. Nalimov, Z.M. Mulchenko, Measurement of Science: Study of the Development of Science as an Information Process (Springfield, Virginia, 1971)

4. J. Mingers, L. Leydesdorff. Eur. J. Oper. Res. 246 (2015)

5. V. Ittipanuvat, K. Fujita, I. Sakata, Y. Kajikawa. JET-M 32 (2013)

6. Enformation, Profil Access, - http://apps.webofknowledge.com.am.enformation.ro/Search.do?product=WOS\&SID=C4YeBq7S3ns9iuWbeK7\&search_mod $\mathrm{e}=$ GeneralSearch\&prID=6f092f57-8546-4990-851f-2ec1e65b0bb5; Accessing date: 14.01.2018

7. VOSviewer - Visualizing scientific landscapes, Retrieved February 8, 2018, from http://www.vosviewer.com/; Accessing date: 08.02.2018

8. M. Rodriguez-Salvador, R. M. Rio-Belver, G. Garechana-Anacabe, PLoS One, 6 (2017)

9. B. Torrisi. Scientometrics. 99, 3 (2014)

10. M. A. Martinez, M. J. Cobo, M. Herrera, E. Herrera-Viedma, Res. Social Work Prac. 25, 2 (2015) 